\title{
Pediatrics in China: challenges and prospects
}

\author{
Yang Liu ${ }^{1} \cdot$ Li-Li Yang $^{1} \cdot$ Si-Yun Xu ${ }^{1} \cdot$ Zheng-Yan Zhao $^{1,2}$
}

Received: 18 October 2017 / Accepted: 12 November 2017 / Published online: 30 January 2018

(c) Children's Hospital, Zhejiang University School of Medicine 2018

China has the largest number of children in the world. The population of children between 0 and 14 years of age was about 227 million in 2015. It is a great and arduous task to ensure the healthy growth of all children in China. Maternal and child health care in China has achieved significant improvements. China has achieved the Millennium Development Goal 4 [1], the mortality rate of children under 5 years of age showed an overall downward trend from 61.0 per 1000 in 1991 to 10.7 per 1000 in 2015 [2]. However, China still faces many challenges, such as the serious shortage of pediatricians, shortage and uneven distribution of medical resources, imbalances among different population groups and different regions.

Shortage of pediatricians is a big problem across China. According to the data of National Health and Family Planning Commission (NHFPC) [3], the number of practicing physicians in pediatrics was about 118,000 , which was 0.53 per 1000 children aged $0-14$ years (Table 1 ). In the developed counties, this number is from 0.8 to 1.5 . The "pediatrician shortage" reflects some common issues suffered by the current medical personnel in China, such as high work pressure, low income and long working hours. In addition, the difficulties and risks faced by pediatricians tend to be greater. The number of outpatient visits per pediatrician was about 2.4 times of that of other physicians in the medical institutions. The average annual number of discharged person was about 2.6 times of that faced by other physicians [3]. The lower income and heavier workload resulted in a high staff turnover rate. During 2011-2014, about 14,310 Chinese pediatricians quitted their job, accounting for $10.7 \%$ of the total number of pediatricians. At the same time, not many

Zheng-Yan Zhao

zhaozy@zju.edu.cn

1 Editorial Office of World Journal of Pediatrics, Children's Hospital, Zhejiang University School of Medicine, Hangzhou, China

2 Department of Children's Health Care, Children's Hospital, Zhejiang University School of Medicine, 3333 Binsheng Road, Hangzhou 310052, China medical students want to become pediatricians. As a result, the number of pediatricians in China only increased by 5000 in the last 15 years, and China has a shortfall of more than 200,000 pediatricians. It is very hard to alleviate the shortage of pediatricians in a short time.

The shortage of pediatric medical resources is another big challenge for China. In 2016, there were only 99 children's hospital in China [3], accounting for about $0.52 \%$ of the total number of hospitals, and more than $70 \%$ of which were located in large- and medium-sized cities. The number of hospital beds for children was 258,000 , accounting for about $5 \%$ of the total number of hospital beds in China.

Imbalance of medical resources in China is particularly prominent when compared with the developed countries and some developing countries in the world. Because of the historic, geographic and economic differences, there are significant differences in allocation of health care resources, utilization of health services and the health level of children between rural and urban areas, or among different regions in China. The patients prefer to visit the hospitals in big cities. So the children's hospitals in the big cities are overcrowded, and it is quite difficult for those hospitals to meet the huge demands in examination, surgery and hospitalization. Pediatric services in rural areas are in more serious shortage. The hierarchical treatment and referral system has not been established throughout the country. Moreover, the basic medical insurance system cannot cover all the children, and the reimbursement ratio of most children's medical insurance is very low.

Birth defect is another serious problem in China. With an increase of the maternal age and the pollution problems, the birth defect rate is increasing. There are about 900,000 neonates with birth defects in China each year, with an overall incidence of $5.6 \%$. The perinatal birth defects increased from 109.79 per 10,000 in 2000 in China and to 153.23 per 10,000 in 2011. In 2000, birth defects account for $12.5 \%$ of the deaths of children, however, in 2011, this number raised to $19.1 \%$. Thus, reduction of the birth defect rates is an important measure to decrease the mortality of children and improve the quality of births in China. 
Table 1 Some facts about the status of pediatrics in China

\begin{tabular}{ll}
\hline Per capita GDP of China, 2015 & 50,251 RMB/about 7500 USD \\
Pediatric population (aged 0-14 years), 2015 & 227 million \\
Births per year, 2015/2016 & 16.6 million/18.5 million \\
Children hospitals, 2014 & 99 \\
Hospital beds for children, 2014 & 258,000 \\
Pediatricians, 2014 & 118,000 \\
Pediatricians per 1000 children aged 0-14 years ${ }^{\mathrm{a}}, 2014$ & 0.53 \\
Perinatal birth defects per 10,000, 2011 & 153.23 \\
Pediatric outpatients, 2014 & $465,663,917$ \\
Pediatric emergency visits, 2014 & $85,741,464$ \\
\hline
\end{tabular}

$G D P$ : gross domestic product

${ }^{a}$ Pediatricians per 1000 children in developed countries: 0.8-1.5
To safeguard the rights and interests of the children and promote the comprehensive development of children, China State Council issued the "Program for the Development of Chinese Children (2011-2020)" in 2011 [4]. The government is increasing financial input into the development of pediatrics, and establishes a stable, institutionalized cost guarantee mechanism, aiming to deliver more efforts to the poor areas and the western areas in China, and reduce the disparities in pediatric service capabilities and quality between urban and rural areas. The fruits of reform and development should be enjoyed by all the children. Children should enjoy more free basic services. Ensuring the safety and health of children is a strategic element to build a harmonious society and improving China's society development. According to the "Opinions on Strengthening the Reform and Development of Children's Health Service" of the NHFPC, by 2020, there will be 2.2 hospital beds per 1000 children. Each township hospitals and community health service institutions have at least one general practitioner to provide standard basic medical services for children.

To solve the shortage of pediatricians, the Ministry of Education of China is actively taking measures to strengthen pediatric medical personnel training, including the deepening of grass-root-oriented training of general practitioners and further expanding the pediatric medical graduate enrollment, which requiring the 38 high-level medical colleges and universities in China to increase the number of postgraduate pediatric enrollment, and strive to reach 10,000 students by 2020 . At the same time, standardized training of pediatric professional residents and pediatrician transfer training is also an important way to complement pediatric medical personnel. During China's 13th 5-year plan (2016-2020) on National Economic and Social Development, China will recruit about 5000 general practitioners each year to provide pediatric medical services in the primary health care institutions, and expand the scope of pediatric resident standardized training. The
NHFPC has officially issued the "Transfer Training Program for the Pediatricians in 2015" to carry out pediatrician transfer training to improve the capacity of pediatric medical services and alleviate the shortage of pediatric physicians. China will recruit more than 30,000 pediatric professional residents by 2020 , and increase the number of pediatricians to more than 140,000.

In summary, serious shortage of pediatricians and disparities of medical resources are the most prominent challenges faced by China. China is trying to solve these problems by strengthening pediatric medical personnel training, improving children's health service system and promoting the reform in the field of children's health service. This process should be in a government-led, whole society-participated manner.

Author contributions Zhao ZY and Liu Y drafted the initial manuscript. All authors reviewed, revised, and approved the final manuscript.

Funding This study was supported by Project for Enhancing International Impact of China STM Journals (PIIJ2-C-31) and Education Department of Zhejiang Province (Y201329546).

\section{Compliance with ethical standards}

Ethical approval Not needed.

Conflict of interest The authors have no financial relationships relevant to this article to disclose.

\section{References}

1. China National Health and Family Planning Commission. The 2015 statistical bulletin on development of China health and family planning career. http://www.nhfpc.gov.cn/guihuaxxs/ s10748/201607/da7575d64fa04670b5f375c87b6229b0.shtml. Accessed 24 Oct 2017 (in Chinese). 
2. Xi B, Zhou C, Zhang M, Wang Y, Xu L. Maternal and child mortality in China. Lancet. 2014;383:953-4.

3. National Health and Family Planning Commission of the People's Republic of China. Press conference: about the pediatric medical service in China (2016). http://www.nhfpc.gov.cn/zhuz/ xwfb/201602/72885a2c88e14b53b25f31cedb76eb80.shtml. Accessed 23 Jan 2018 (in Chinese).
4. The State Council of the People's Republic of China. China Maternal and Child development Compendium (2011-2020). (2011). http://jtjy.china.com.cn/2013-03/06/content_57803 60.htm. Accessed 24 Oct 2017 (in Chinese). 